\title{
Oral Lichen Planus- a Mucopigmentory Disorder
}

\author{
Neha Aggarwal ${ }^{* 1}$ and Sumit Bhateja ${ }^{2}$ \\ Department of Oral Medicine \& Radiology, Manav Rachna Dental College \& Hospital, Faridabad, India \\ ${ }^{2}$ Reader Dept of Oral Medicine and Radiology, Manav Rachna Dental College, India
}

Received: May 19, 2018; Published: May 29, 2018

*Corresponding author: Neha Aggarwal, Department of Oral Medicine \& Radiology, Manav Rachna Dental College \& Hospital, House no 478, R/L, Near Old Sawhney, Hospital Faridabad, Haryana, India

\begin{abstract}
Lichen planus (LP) is a distinctive entity with prototypic "lichenoid" papules that show distinctive color and morphology, develop in typical locations, and manifest characteristic patterns of evolution. Microscopic features, similar to the gross morphology, are distinctive although the microscopic pattern of inflammation and skin response is shared by several dermatome.
\end{abstract}

Keywords: Oral Lichen planus; Lichenoid reaction; Treatment

\section{Introduction}

Lichen planus (Greek leichen, "tree moss"; Latin planus, "flat") is a unique, common inflammatory disorder that affects the skin, mucous membranes, nails, and hair [1]. The term lichenoid reaction is the histologic description used to capsulize the pathologic characteristics of skin diseases resembling Lichen Panus. The term lichen ruber planus (Latin ruber, "red" or "ruddy") has been used to denote the distinctive color of the lesion, but this terminology has been largely abandoned. The four Ps-purple, polygonal, pruritic, papule-is the abbreviation often used to recall the constellation of symptoms and skin findings that characterize Lichen Planus [2]. It occurs throughout the world, in all races. It may be familial in $1 \%$ to $2 \%$ of cases. It appears in men at a constant rate from the early 20 s through the 60 s, whereas in women the rate of new cases continues to increase with increasing age, reaching a peak in the 60s.30lder and resolving lesions are often hyper pigmented. There is a predilection for the flexor wrists, trunk, medial thighs, shins, dorsal hands, and glans penis. Koebner's phenomenon occurs in Lichen Planus and pruritus is often prominent $[2,3]$.

Children represent only $4 \%$ of cases of LP, and the lesions are often atypical. Linear or segmental patterning, prominent follicular involvement, significant nail changes with deformities, and a long course are common in children. Children with lichen planus often have affected family members. Children from the Indian subcontinent appear to have lichen planus more frequently. OLP is a syndrome diagnosis, i.e. based on the presence of several clinical and histopathological criteria. Thus, the diagnostic approach is best described as a method of pattern recognition both clinically and histologically [4]. In 1978 a clinical and histopathological definition of lichen planus was formulated by WHO as: "the disease commonly affects the oral mucosa, and lesions may occur in the mouth in the absence of skin lesions. Mucosal lesions are usually multiple and often have a symmetrical distribution. They commonly take the form of minute white papules which gradually enlarge and coalesce to form either a reticular, annular, or plaque pattern. A characteristic feature is the presence of slender white lines (Wickham's striae) radiating from the papules. In the reticular form there is a lace like network of slightly raised gray white lines, often interspersed with papules or rings. The plaque form may be difficult to distinguish from leukoplakia, but in lichen planus there is usually no change in the flexibility of the affected mucosa. In some patients the lesions are atrophic, with or without erosions. Oral lesions of lichen planus may also include bullae, but these are rare .The histopathologic features of lichen planus are characteristic. There is usually a keratinized layer, and this may be either orthokeratinized or parakeratinized. If keratinization is normally found at the affected site, then the keratinized layer is thickened. If the site is normally nonkeratinized (for example, buccal mucosa), then the keratinized layer in the lichen planus lesion may be very thin. Similarly, if there is normally a stratum granulosum this will be thickened. If there is normally no stratum granulosum then granular cells may be present in small numbers. The 'saw tooth' appearance of the rete processes that is a common feature of skin lesions is less frequently seen in the oral mucosa. The thickness of the epithelium varies, and atrophy is often seen. Civatte (colloid) bodies may be present in the region of the basal-cell layer, lying either in the epithelium or within the superficial part of the connective tissue. These are rounded or lobulated acidophilic structures which sometimes contain a py- 
knotic nucleus or nuclear fragments. The changes in the basal cell layer often include 'liquefaction degeneration', and there may be a narrow band of eosinophilic material in the position of the basement membrane. There is a well-defined zone of cellular infiltration that is confined to the superficial part of the connective tissue (lamina propria), and the infiltrate consists mainly of lymphocytes except in the vicinity of an erosion" [5].

At first glance, the definition of OLP seems to result in a well described entity. However the process by which the pathologist makes a diagnosis is inherently subjective. The purpose of diagnostic process is to make it possible to decide on treatment and prognosis. For instance, studies on the possible premalignant character of OLP are less meaningful, and may even be confusing and inconsistent if a precise diagnosis of OLP cannot be made. Confirmation of the clinical diagnosis of OLP by means of histologic study of biopsy specimen is generally advised [6].

\section{History}

The disease was first described in 1869 by Erasmus Wilson as lichen planus [7]. Among the numerous cases in his detailed study, eruptions were noted on the buccal mucosa, tongue, and pharynx. The oral lesions in LP were further noted and described by Unna (1882). Thibierge (1885) first described the oral lesions systematically. He felt that in most cases the lesions occurred on the buccal mucosa and the tongue, with certain differences in appearance. The lichen ruber described by von Hebra (1890) was not LP, although presenting certain similar features. Habra's description is now considered to be that of pityriasis rubra. At this time it was felt that the oral lesions were merely an accompaniment to the generalized skin eruption. It was Audrey (1894) who pointed out that the oral lesions of lichen planus could occur in the absence of skin lesions. Wickham, a French physician, wrote a paper on a pathognomonic signs of "Wilson's lichen" in 1895. He described the fine white grayish lines that could be seen on the skin papules of the disease (later known as Wickham's striae) but he did not mention the clinical appearance of the oral lesions [7]. Crocker (1900) noted white lines and white spots on the buccal mucosa and symmetric plaques on the sides of the tongue in several cases. Poor (1905) was first to describe the formation of "cavities" in lichen planus of the mucosa, corresponding in character to sub epithelial bullae and characterized by exudation from surrounding blood vessels. Dubreuilh (1906) stated that aside from the numerous cases of lichen planus involving both skin and mucosa, the involvement of oral mucous membrane alone was more common than involvement of the skin without mucosal lesions. From a study of biopsies from lesions of the tongue and buccal mucosa, he felt that histologically these lesions were in all points comparable to those of the skin. Furthermore, because of the ease of oral biopsy, Dubreuilh suggested this as a diagnostic aid [8]. Lieberthal (1907) who first described the oral manifestations of LP in the American literature realized that the lesions of the tongue were different from those of buccal mucosa. The tongue usually had irregular oval or circular plaques, while the cheek usually had papules, oval, or circular plaques, most frequently streaks or criss-crossing linear projections. Black MM (1986) quoted that lesions may be restricted to either location or may also occur on both skin and oral mucosa in one patient. About 90\% patients who have OLP also have skin lesions [9]. Sapp JP defined lichen planus as "A skin disease common within the oral cavity, where it appears as either white reticular, plaque, or erosive lichen with a prominent T-lymphocyte response in the immediate underlying connective tissue" [10].

\section{Etiology}

Virtually all diseases result from the interplay of host life style and environmental factors and OLP is not an exception. Cell mediated immunity appears to play major role in the pathogenesis of OLP, possibly initiated by endogenous or exogenous factors in persons with a genetic predisposition to the development of LP [11].

A. Genetic Factors From a study of 108 cases of OLP, including drug induced lichenoid reactions, it was indicated that lichen planus is a predetermined condition or diathesis, perhaps genitically based, rather than simple cause-and-effect disorder [12]. Only weak associations have been demonstrated between HLA antigens and LP. An increase in HLA-DR9 and Te 22 antigens has been noted in Chinese patients.

B. Dental Materials No evidence of any association of OLP with dental restorative materials was found in most patients. However, contact with or proximity to restorations involving amalgams or other materials causes some lichenoid reactions. These reactions are presumably due to allergic or toxic reactions to compounds released or generated, the Koebner phenomenon, or possibly plaque accumulated on the surfaces of the restorations. Some lesions resembling OLP may occur in direct relation to amalgam restorations and some of these oral lesions may improve after substitution of the amalgam by other materials though this is often not the case with gingival lesions. Patch test positivity was found against mercury in $39.6 \%$ of 48 patients. Of those who had a positive patch test to mercury, $94.7 \%$ showed regression of lesions after removal of amalgams, but even $82.6 \%$ of those who showed no reaction to mercury on patch testing also showed regression after removal of amalgams. OLP patients show a higher correlation with delayed hypersensitivity to dental materials than a control population; however, a cause-and-effect relationship could not be substantiated [13]. Contact allergy to mercury was suggested as possible etiologic factor [14]. There are also reports of OLP in relation to gold restorations, composite restorations and cobalt in a restoration. However, fillings only should not be the only factor involved in the cause of these lesions [15].

\section{Drugs}

The possible association of drugs with lesions similar to OLP was noted when quinacrine and mepacrine used as antimalarials during World War II, were seen to cause lichenoid lesions. Hakala (1986) reported gold salts causing a range of mucocutaneous lesions, of which oral lichenoid lesions may be the first. The drugs now most commonly implicated in OLP like lesions are the Non-steroidal Anti-inflammatory drugs and the Angiotensin-Converting Enzyme inhibitors. Other drugs known to cause lichenoid eruptions include thiazides, diuretics, penicillamine, beta-blockers, 
quinine and quinidine, para-amino salicylic acid, phenothiazines, carbamazepine, allopurinol, lithium, lorazepam, ketoconazole, streptomycin, isoniazid, metopromazine, levopromazine, amiphenazole, pyrimethamine, levamisole, beta-blocking agents, cinnarizine, f1unarizine, gold, cyanamide and many others. The list of drugs implicated lengthens almost weekly and, interestingly, includes a number of agents which have also been used in the therapy of lichen planus, particularly dapsone, levamisole, tetracycline, and interferon.

\section{Infectious Agents}

Jacob and Helmbold (1933) suggested OLP to be related to bacteria such as a Gram-negative anaerobic bacillus. Lichenoid reactions have been seen in syphilis, chronic bladder infection, and intestinal amebiasis. Studies have shown an increased prevalence of candida species-in both mycological and histological studies of OLP. The frequency of OLP patients with candidal infection, however, was the same as in healthy dentulous adults generally [16]. Although, in some of the candida positive patients, antifungal medications appeared to be useful [17]. The involvement of viral agents in OLP has been suggested. Lichenoid lesions may be seen in HIV infection. Human papilloma viruses (HPV) have been found in lesions of LP but any causal role remains speculative. The finding of HPV-DNA in many of the erosive OLP cases using Southern blot hybridization with P-labeled HPV-DNA probes indicate a high prevalence of HPV in erosive OLP affected mucosa [18] Low רgrade and/ or persistent infection of epithelial cells with herpes viruses may be a possible etiologic factor in LP. Interestingly, humoral responses to EBV appear to be altered in OLP.

A. Autoimmunity The possible contribution of autoreactivity to the pathogenesis of OLP has been suggested on the basis of studies demonstrating changes in T-lymphocytes in the peripheral blood, including a depressed number of CD4+ and CD45RA+ cells in OLP patients.

I. Chronic Liver Disease: Rebora, 1981 was the first to report five cases of erosive LP associated with severe liver disease. The liver diseases that seem to be most strongly related to OLP are chronic liver diseases, especially chronic active hepatitis and primary biliary cirrhosis. El-Kabir (1993) did not find a significant association between OLP and chronic liver diseases. A study of a large number of patients with chronic liver disease found only a very low prevalence of LP (Golding 1973).

II. Pawlotsky (1994) stated that Hepatitis B virus (HBV) could be a causal agent of LP-associated liver disease, since several reports suggested a relationship between HBV and LP.

Other autoimmune diseases: Associations of LP with several different autoimmune diseases like Alopecia areata, Dermatitis herpitiformis, Dermatomyositis, Hashimoto's thyroiditis, Hyperthyroidism, Lupus erythematosus, Morphea, Myasthenia gravis, Pemphigus foliaceus and vulgaris, Pernicious anemia, Rheumatoid arthritis, Sjogren's syndrome, Scleroderma and Vitiligo have been documented.
III. Immunodeficiencies Cutaneous LP is more strongly associated with defects of T-cell function such as thymoma or HIV than it is with humoral immunodeficiencies, though LP has been observed in hypogammaglobulinemia. OLP may also be seen in HIV associated diseases. There is no consistent alteration in the serum level of immunogloblins in OLP. Varied levels of IgA, IgG, IgM, IgE and IgD have been observed in LP, and serum levels of complement components are normal.

IV. Food Allergies A small minority of patients with OLP or lichenoid lesions have been shown to react to certain foods and some to food additives such as cinnamon aldehyde.

V. Stress Stress has been widely held to be an important etiologcal factor in OLP. A statistically significant difference was indeed found in the psychological profiles of patients affected by OLP as compared with those of controls. In a study on psychiatric disturbance in patients with OLP, most of the patients had subjective feeling that the clinical appearance of OLP, including discomfort, became worse during times of mental stress [16]. Studies showed no statistically significant association between OLP and either anxiety or depression $[19,20]$.

VI. Habits Smoking has been suggested to be an etiological factor in some Indian communities. Betel nut chewing is also more prevalent in Indian patients with OLP than in those without the habit (Daftary 1980).

VII. Diabetes and Hypertension The high incidence of diabetes mellitus (DM) in the patients with OLP supports the hypothesis that DM may be related to pathogenesis of OLP [21]. Several studies have now shown only low prevalence of OLP in large groups of diabetic subjects such that the suggested association may be only coincidental or caused byanti-diabetic or other drugs. Nevertheless, in diabetics with OLP, there may be a higher prevalence of lingual involvement and of erosive lesions. Blood pressure, when evaluated in LP, appears as an independent variable with no significant correlation. Grinspan described seven cases of OLP associated with diabetes and hypertensions (Grinspan 1966), a triad which became known as Grinspan's syndrome [21].

VIII. Malignant Neoplasms Lichenoid lesions have been observed on the skin and/or mucosae of patients affected by a range of different neoplasms like breast cancer, metastatic adenocarcinoma, retroperitoneal sarcoma, stomach cancer, thymoma, Castleman's tumor, craniopharyngioma, pituitary adenoma and Non-Hodgkin's lymphoma. The clinical presentation of LP associated with malignancies most commonly seems to be the bullous type, sometimes lichen planus-pemphigoides, and on some occasions it can clinically mimic paraneoplastic pemphigus.

\section{Bowel Disease Lundstrom}

X. (1982) reported the association between ulcerative colitis and LP. Other bowel diseases occasionally described con- 
comitant with LP include Coeliac disease and Crohn's disease, though others have not found these associations.

XI. Miscellaneous Associations LP has occasionally been associated with other conditions, including Psoriasis, Lichen sclerosis, Urolithiasis, Mesangioproliferative glomerulonephritis, Erythema dyschromicum and Turner's syndrome with endocrinopathies.

\section{Pathogenesis}

The data fundamental to the pathogenesis of LP has been given by several workers. The etiopathogenesis of OLP appeared to be complex, with interactions among the genetic, environmental and life style factors, but much has now been clarified about the mechanisms involved, and interesting new associations such as with liver disease have emerged. There are extensive data to suggest that immunological mechanisms, mainly cellular, are fundamental to the pathogenesis of LP. Epidermotropic, MHC- specific, autoreactive T-cells can produce a histopathological picture indistinguishable from that of LP when injected into the footpads of syngenic mice, and patients with chronic graft-versus-host disease may develop cutaneous and oral lesions clinically and histopathologically similar to those of LP [22]. Also therapies that suppress cell mediated immunity, e.g., cyclosporin and etritinate both reduce the lymphocyte infiltrate and induce clinical improvement. A lymphocytic immunological reaction against the epithelial basal cells is the mechanism responsible [23]. It was suggested that the pathogenesis of LP involved antigen presentation by langerhans cells (LC) to helper-T cells, activated $\mathrm{T}$ cell elaboration of interleukin-2 (IL-2), interferon- $\gamma$ and tumour necrosis factor $\beta$ with subsequent recruitment of more $\mathrm{T}$ cells and induction of keratinocytes to produce cytokines. Cytotoxic T cells, lymphotoxins, and cytokines may mediate basal cell liquefaction and other keratinocyte damage. It was documented that in early lesions of lichen planus, the T cells of the dermal infiltrate were CD4 +/DR+/ activated T-cells of the helper phenotype, some closely opposed to macrophages and langerhans cells. Later, T-cells infiltrating the epidermis were of the CD8+ cytotoxic/ suppressor phenotype.

Hypothesis for antigen presentation and T-cell activation in OLP. Initially, the CD8+ T-cell antigen receptor engages a specific foreign antigen (Ag 1) in the context of MHC class I on the basal keratinocyte target cell in OLP [1]. The CD8+ T-cell may then seek CD4+ T-cell confirmation by expressing the hypothetical "request cytotoxic activity" (RCA) cell surface molecule [2]. The CD4+ T-cell expresses the hypothetical "RCA receptor" (RCA R) [4], but only following CD4+ T-cell antigen receptor engagement of a related foreign antigen (Ag 2) in the context of MHC class II on the antigen-presenting cell (basal keratinocyte or Langerhans cell in OLP) [3]. Ligation between RCA and RCA R in combination with co-stimulatory signals from the MHC class II+ antigen-presenting cell (e.g., CD40, CD80, and IL-12 binding CD154, CD28, and IL-12 R, respectively, on the CD4+ T-cell) initiates Th1 differentiation of the CD4+ T-cell that then secretes IL-2 and IFN-g [5]. Receptors for IL-2 and IFN-g are expressed by the CD8+ T-cell, but only following (i) specific engagement of the CD8+ T-cell antigen receptor in the context of
MHC class I and/or (ii) ligation between RCA and RCA R. The CD4+ Th1 cytokines (IL-2 and IFN-g) are detected by the CD8+ T-cell and interpreted as confirmation to proceed with target cell (basal keratinocyte) lysis. Keratinocyte activation by

\section{i. the CD4+ or CD8+ T-cell following receptorantigen- MHC trimerization or}

ii. exogenous agents such as viral infection, bacterial products, mechanical trauma, systemic drugs, or contact sensitivity up-regulates keratinocyte cytokine and chemokine secretion [6] that promotes lymphocyte extravasation and directs lymphocyte migration into the site of the developing OLP lesion. Basement membrane disruption in OLP may be mediated by mast cell proteases directly or indirectly via activation of T-cell-secreted MMP-9 [24].

\section{Epidemiology \\ Prevalence}

The prevalence of lichen planus in general population is of the order of 0.9 to 1.2 per cent [25]. Hellier (1940) in a study of environment and skin diseases reported that OLP occurred between 0.1 to 1.25 percent of the dermatological out patients. Arndt (1971) in a large survey of 676,376 dermatological out patients in various hospitals in United States of America revealed a prevalence of 0.442 percent while Shklar G (1972) reported that the prevalence of OLP in routine dental clinic was around 0.6 percent [26]. In another study the annual age-adjusted incidence rate was 2.1 and 2.5 per 1000 among men and women respectively [27]. Neville BW et al in their text book mentioned a prevalence of oral lichen planus between 0.1 to 2.2 percent among the general population [28].

I. Age: It is mentioned that Lichen planus is a disease of middle aged and elderly with ages ranged from 30 to 70 yearswhile a prospective study of 570 OLP patients showed mean age of 52 years [29]. According to another study the age range for males with lichen planus has been about a decade lower than for females with lichen planus. Neville BW et al. [28] mentioned that most patients with lichen planus are middle aged adults and it is rare for children to be affected. According to Shafer's 2006it predominantly occurs in adults older than 40 years, although younger adults and children can be affected.

II. Sex: Lichen planus affects both the sexes, although occasionally some survey has suggested a male predominance but majority of the studies suggested a female predominance. In the clinical aspects of OLP a high incidence of $66 \%$ among females was reported when compared with males. Other follow up studies on OLP observed an incidence of 62.6 percent in females. 3:2 female predominance over male while according to Shafer's 2006 is 1.4:1.Site: Study on variation in course of lichen planus reported that 90 percent occurred on the buccal mucosa, 60 percent on tongue and 4 percent on gingiva but did not report the involvement of other areas.29It was stated that 90 percent of the cases of OLP occurred on buccal and labial mucosa, 27 percent on tongue, 23 percent on gingiva, and 7 percent on the palate [30]. 
III. Geographic distribution: LP patients were recorded in all races. Prevalence of LP in American blacks was found to be 0.29 percent. In Hungarians it was found to be 0.6 percent. Mehta (1971) mentioned LP prevalence ranged from 0.1 to 1 percent.

\section{Clinical Features}

In 1968 Andreasen JO examined 115 cases of OLP and recognized six clinical types: reticular, papular, plaque like, atrophic, erosive and bullous lesions. Tyldesley WR (1974) described OLP in three different forms i.e. non-erosive, minor erosive and major erosive lichen planus. In non-erosive lichen planus the clinical picture presented by the patient was of milky white streaks over the oral mucosa arranged in linear, reticular, papular or confluent plaque like manner and lesions were usually asymptomatic with patients complaining of roughness of the mucosa. The clinical picture presented by the patients in minor erosive lichen planus was that of shallow erosions of the oral mucosa associated with milky white areas as seen in case of non-erosive lichen planus and patients feel uncomfortable on contact with spicy or acidic food [30]. In major erosive lichen planus the patient had usually widespread ulceration of the buccal and labial mucosa and tongue. The ulcers were painful with a peculiar glaze and a well demarcated edge.

Cutaneous lesions are bilaterally symmetrical and found most often on the flexor surfaces of the wrists and forearms, the inner aspect of the knees and the sacral areas. The typical lesion is a small red, purple or brown flat-topped polygonal papule covered with a glistening scale. The papule may coalesce and form patterns. Oral lesions are characterized by small whitish hyperkeratotic papules whose arrangement makes up the typical linear, annular and reticular thread like patterns. Other oral lesions have been described as plaque like, papular, pigmented, verrucous or hypertrophic, atrophic, erosive or ulcerative and vesiculo-bullous. In most forms, however, some evidence of characteristic radiating striae can be seen. Nail changes with lichen planus are not common and that too without other cutaneous manifestations are considered rare.

A study involving 611 patients with OLP found that reticular type was the most common form (92 percent) and was present in almost all patients while atrophic was the second most common form (44 percent) [31]. The various clinical types had somewhat different courses. Papular affections were seen mainly in the initial phase and had a transitory course. Although ulcerative lesions are more persistent; but they also generally showed a short term course. The atrophic form was fluctuating with many remissions and new-established affections. Initial popular lesions were associated with ages below 50 years and atrophic lesions with ages above 60 years. Plaque type lesions were seen more in tobacco smokers at the onset of OLP. No other correlation was found between different clinical forms and clinical course with various factors as age, sex, general disease, medication and tobacco smoking [32].

Reticular, plaque like or papular type is often quite painless and may be discovered by "accident" by the examining dentist. The patient may have just noticed a "roughness" about which she or he had not sought advice. Erosive, atrophic or bullous oral lichen planus is nearly always painful and merits treatment. Most of OLP patients were asymptomatic and appears as hyperkeratotic plaques or striae. Inflammatory exacerbations result in erosive or bullous forms, with symptoms ranging from mild discomfort to severe burning pain [33]. Bilateral lesional distribution is a key diagnostic feature, and unilaterality is a detractor and actually renders true LP less likely. The lesion appears as keratotic papules or confluent, lacy, striated plaque on an erythematous background. The classic hypertrophic LP is generally painless; however, symptoms varying in degree often attend erosive, ulcerative, or atrophic variants [34]. The clinical manifestations of OLP lesions range from the less severe reticular and atrophic form to the more acute and painful erosive and rarer bullous form [35].

Lichen planus was quite frequently an indolent, painless lesion that may sometimes be present in the patient's mouth and recognized only during the routine dental examination. It has slightly elevated, fine, whitish lines known as Wickham's striae that produce either a lace like lesion, or radiating lines or annular lesions. In papular form, the lesions were usually $0.5 \mathrm{~mm}$ to $1 \mathrm{~mm}$ whitish, elevated lesion and usually seen in well keratinized areas of the oral mucosa and the bullous form of lichen planus was rare erosive lesion develop as a complication of atrophic processes when the thin epithelium was abraded or ulcerated. OLP appears to be well defined and completely described entity according to WHO (1978) definition. However it is known that process by which a pathologist makes a diagnosis is inherently subjective, so it is important to check histologic variability. Onofre et al. studied the correlation between clinical and histopathologic diagnoses in 45 patients with leukoplakia and OLP and found a clinicopathologic discrepancy in a quarter of these lesions [36]. In a study performed to check intraexaminer and interexaminer reliability in the diagnosis of oral epithelial dysplasia, it was found difficult to achieve accurate reproducible agreement [37]. Another study about observer variability in the histologic assessment of oral premalignant lesions reported the interobserver agreement rates in the range of $49 \%$ to $69 \%$. The calculated kappa values were in the range of $27 \%$ to $45 \%$, showing poor to moderate agreement between the pathologists [38].

\section{Histopathologic Features \\ Light Microscopic Features:}

Darier J (1909) was the first person to describe the classic histopathological features of LP as hyperparakeratosis/ hyperorthokeratosis, acanthosis, thickening of granular cell layer, basal cell liquefaction, "saw" tooth rete ridges and band like dense inflammatory cellular infiltrate in the upper lamina propria. OLP was described basically as a mesenchymal disease with secondary epithelial changes. Ellis FA (1967) found that occasionally small areas of artifactual separation between the epidermis and the dermis, known as Max- Joseph space, seen. In some instances, the separation occur in vivo and subepidermal blister form (vesicular lichen planus). These vesicle forms as a result of extensive damage to the basal cells [39]. Tyldesley WR (1974) mentioned a well-defined band of 
inflammatory cells predominantly lymphocytes, lies below, but separate from dermal-epidermal junction. Epithelium overlying this dermis may show a variety of reaction from atrophy to acanthosis with either hyperparakeratosis or hyperorthokeratosis. Hyperparakeratosis is common in oral lesions compared to skin lesions i.e. in 86 percent of OLP patients. Very often rete pegs are flattened, and although a "saw tooth" appearance of rete pegs is often described. It is by no means evident in all lesions. In a retrospective study of 112 cases, it was found that mononuclear infiltration beneath and adjacent to the epithelium, parakeratosis and degeneration of the basal layer of epithelium were the consistent features [40].

It was believed that Wickham's striae are caused by a focal increase in the thickness of the granular layer and of the total epidermis. The classical histologic change in lichen planus include hyperplasia and/or hyperkeratosis in reticular, plaque like or papular form, or atrophy or erosion, liquefaction degeneration of basal epithelial cells, saw tooth rete ridges, occasional civatte bodies, band like inflammatory infiltrate in tissue immediately subjacent to the epithelium. Retrospective examination of records of 250 cases of OLP in the period of 1996-1998 found one case in which part of the subepithelial connective tissue was replaced by granular cell proliferation. They conclude that the presence of granular cells might rather have been a reactive phenomenon triggered by the inflammatory infiltrate or a granular cell tumor [41]. The degeneration of the basal keratinocytes and disruption of the anchoring elements of the epithelial basement membrane and basal keratinocytes (e.g hemidesmosomes, filaments, fibrils) weaken the epithelial-connective tissue interface. As a result, histologic cleft (i.e. Max-Joseph space) may form, and blisters on the oral mucosa (bullous lichen planus) may be seen at clinical examination.

\section{Dysplastic Features in Oral Lichen Planus}

Kaugars EG and Sristy AJ (1982) studied dysplastic or carcinomatous transformation of oral lichen planus. They reported that epithelial dysplasia could be found up to 0.23 percent in oral lichen planus but did not mention the dysplastic features. They also stated that it was difficult to determine whether the epithelial dysplastic feature was in response to lymphocytic infiltration or the epithelium itself was neoplastic in nature [42]. In another study of epithelial dysplasia in one hundred cases of OLP; 87 percent of cases had the presence of more than one layer of cells having a basaloid appearance, 60 percent nuclear hyperchromatism, 45 percent enlarged nucleoli, 33 percent loss of polarity of the basal cells and 27 percent keratinization of single cell in the spinous layer. It was also reported that the complete absence of dysplastic features was in only 6 percent of material, while in 24 percent, a total number of 5 or more dysplastic changes were recorded (among 12 criteria listed by WHO collaborating reference centre for oral precancerous lesions) [43]. In a study of 722 patients of OLP epithelial dysplasia was observed in 4 percent of cases [44].

\section{Electron Microscopic Feature of Oral Lichen Planus}

Ultrastructural studies on vesicular lesions of lichen planus reported that the basal keratinocytes showed cytolysis and the blister cavity was situated below the spinous layer. The cellular changes observed in the epithelium were in no sense specific for the condition, although the overall picture was quite characteristic, suggesting a disease process involving all layers of epithelium and of more complex nature than an abnormality of keratinization. Widening of intercellular spaces in the basal layer and lower stratum spinosum, as well as acantholytic changes in the basal layer, separation of the basal lamina from the basal cells and fragmentation of the basal lamina is present. Desmosomes between many basal cells as well as hemidesmosomes between basal cells and basal lamina are lost and the basal lamina is separated from the epithelium to become thick, irregular and fragmented. Tonofilaments in the cell also had lost orientation at the desmosomal region and their numbers were greatly reduced.

\section{Immunofluorescence Studies of Lichen Planus}

Immunofluorescence studies on lichen planus reported IgM immunoglobulin and B1C/B1A complement deposited at basement membrane zone. Presence of fibrin deposition at the mucosal-submucosal junction within vessels and cytoid bodies was found to be highly characteristic of LP, but not specifically diagnostic. Morphologically identical deposits were also seen in lupus erythematosus (LE) [45]. It was also concluded that Ig deposits are characteristic feature of oral lesions of discoid LE and systemic LE and only rarely occurs in OLP and leukoplakia [46]. Serologic investigation aimed to identify an IgG antibody that under immunofluorescent staining produced an annular fluorescence of basal cells and that has been referred to as basal cell cytoplasmic antibody. In diagnosis of drug induced lichenoid reaction these may be useful adjunct to clinical and histologic investigation [47].

\section{Treatment}

Several therapeutic agents have been investigated for the treatment of oral lichen planus (OLP). Among these are corticosteroids, retinoids, cyclosporine, and phototherapy, in addition to other treatment modalities. A systematic review of clinical trials showed that particularly topical corticosteroids are often effective in the management of symptomatic OLP. Systemic corticosteroids should be only considered for severe widespread OLP and for lichen planus involving other mucocutaneous sites. Biopsy and histological confirmation in cases of OLP is recommended where there is uncertainty about the diagnosis or where the lesions are clinically atypical (to exclude the presence of dysplasia or malignancy). Routine HCV testing of patients with OLP is not indicated. Topical corticosteroids (with or without topical antimycotics) are the first-line treatment; currently, there is insufficient evidence regarding different dosages, formulations, or modes of delivery of topical steroids (e.g., paste, spray, mouthwash) to make an evidence-based recommendation about which is best. Systemic corticosteroids (with or without topical antimycotics) are the first-line treatment only for severe, widespread OLP and for lichen planus involving other mucocutaneous sites (e.g., vaginal/vulval LP) resistant to topical therapies. Topical retinoids should be considered only as second-line therapy for OLP; systemic retinoids are not recommended. 


\section{Various treatment options [48]:}

\section{A. Corticosteroids Topical}

i. Betamethasone phosphate

ii. Betamethasone valerate

iii. Clobetasol propionate

iv. Fluocinolone acetonide

v. Fluocinonide

vi. Fluticasone propionate

vii. Hydrocortisone hemisuccinate

viii. Triamcinolone acetonide

\section{B. Systemic}

i. Prednisone

ii. Methylprednisolone

C. Retinoids Topical

a. Fenretinide

b. Isotretinoin

c. Tazarotene

d. Tretinoin

D. Systemic

a. Acitretin

b. Etretinate

c. Isotretinoin

d. Temarotene

e. Tretinoin

E. Immunosuppresive agents

a. Azathioprine

b. Cyclosporin

F. Others

i. Amphotericin A

ii. Basiliximab

iii. Diethyldithiocarbamate

iv. Dapsone

v. Doxycycline

vi. Enoxaparin

vii. Griseofulvin

viii. Hydroxychloroquine sulphate

ix. Interferon

x. Levamisol
xi. Mesalazine
xii. Phenytoin
xiii. Photopheresis
xiv. Psychotherapy
xv. PUVA
xvi. Reflexotherapy
xvii. Surgery
xviii. Tacrolimus
xix. Thalidomide

\section{G. Drug Therapy}

Drug therapy is the most common method for the treatment of OLP. Different drugs have been used for treatment of OLP including immunosuppressives, retinoids, and immunomodulators. Drugs are used in two forms, topical or/and systemic.

\section{H. Topical Drug Therapy}

Topical drug therapy is a method of treatment in which drugs are applied directly to the part being treated (e.g., skin, eyes, or mucosa).Various kinds of drugs are used in topical form for treatment of OLP including corticosteroids, immunosuppressives, retinoids, and immunomodulators.

\section{Topical Steroid Therapy}

High-potency topical corticostroids in an adhesive medium appear to be the safest and most effective treatment of OLP. For topical applications, we usually use them as gel, oral paste, or solution. Triamcinolone has been tried for the treatment of OLP. A number of investigations have determined the efficacy of triamcinolone acetonide $0.1 \%$ suspension in the treatment of OLP. Fluocinolone acetonide gel $0.1 \%$ and fluocinolone acetonide $0.1 \%$ in Orabase and Clobetasol propionate $0.05 \%$ ointment are also used. Intralesional injections of hydrocortisonedexamethasone triamcinolone acetonide and methylprednisolone have been used in the treatment of oral LP.

\section{J. Topical Retinoid Therapy}

Retinoids are metabolites of vitamin A. They have been noted to have antikeratinizing and immunomodulating effects. Isotretinoin gel $0.1 \%$ has also been suggested as an alternative to topical corticosteroids in the management of OLP. Temarotene is a retinoid analogue which is also effective. An oral low dose of tretinoin (all-trans-retinoic acid) has been used in recalcitrant oral LP with complete and partial remissions and without marked side effects.

\section{K. Analgesics}

For symptomatic therapy, the use of a variety of topical analgesics is recommended. Diphenhydramine elixir as mouthwash and xylocaine gel can be safely used alongside other therapeutic agents.

\section{Tacrolimus}

Tacrolimus is a potent immunosuppressive agent, inhibiting T-cell activation.This drug used topically can control symptoms 
and significantly improve refractory erosive oral LP. Tacrolimus ointment $0.1 \%$ is welltolerated and appeared to be effective in erosive oral LP that did not respond to topical steroids.

\section{Ultraviolet Irradiation}

Photo chemotherapy with 8-methoxypsoralen and long-wave ultraviolet light (PUVA) has been used successfully in the treatment of skin lesions and cutaneous LP. It was first used in the treatment of recalcitrant oral LP. Photo chemotherapy may be useful for severe forms of erosive oral LP that do not respond to conventional treatment.

\section{N. Levamisole}

Levamisole is used as animmunomodulator in oral LP. The combination oflevamisole and Chinese medicinal herbs can achieve complete remission more than either therapy given alone. Combined therapy with a low dose of systemic steroid may also be helpful in the control of severe erosive oral LP.

\section{Antimalarials}

Hydroxychloroquine sulfate, chloroquine phosphate can be used for treatment of oral lichen planus.

\section{P. Dapsone}

Dapsone has been used in the treatment of erosive oral LP. It should be considered in resistant cases, particularly when severe erosive lesions are present. Adverse effects such as haemolysis and headache can occur. Generally, the use of dapsone in the treatment oral LP is precluded.

\section{Q. Phenytoin}

It is also effective in treatment of oral lichen planus.

\section{R. Surgery}

Surgical excision has been recommended for isolated plaques or nonhealing erosions because it provides excellent tissue specimens for histopathologic confirmation of diagnosis and may cure localized disease. Cryosurgery has been used successfully in cases of erosive oral LP resistant to most treatment modalities. Free soft-tissue grafts can be used for localized areas of erosive oral LP.

\section{S. Laser}

The $308 \mathrm{~nm}$ excimer laser has been used as a possible and additional method in the treatment of OLP. Treatments are painless and well tolerated. Clinical improvement has been achieved in most patients. Excimer $308 \mathrm{~nm}$ lasers could be an effective choice in treating symptomatic OLP.

\section{References}

1. Al-Hashimi I, Schifter M, Lockhart PB, Wray D, Brennan M, et al. (2007) Oral lichen planus and oral liche-noid lesions: diagnostic and therapeutic considerations. Oral Surg Oral Med Oral Pathol Oral Radiol Endod 103 Suppl: S25: e1-e12.

2. Sugerman PB, Savage NW, Zhou X, Walsh LJ, Bigby M (2000) Oral lichen planus. Clin Dermatol 18: 533-539.

3. Van der Meij EH, Schepman KP, Plonait DR, Axell T, van der Wall I (2002) Interobserver and intraobserver variability in the clinical assessment of oral lichen planus. J Oral Pathol Med 31(2): 95-98.
4. Kramer IRH, Lucas RB, Pindborg JJ, Sobin LH (1978) Definition of leukoplakia and related lesions: an aid to studies on oral precancer. Oral Surg Oral Med Oral Pathol 46(4): 518-539.

5. Van der Meij EH, Reibel J, Slootweg PJ, van der Wall JE, de Jong WFB, et al. (1999) Interobserver and intraobserver variability in the histologic assessment of oral lichen planus. J Oral Pathol Med 28(6): 274-277.

6. Van der Meij EH, van der Wall I (2003) Lack of clinicopathologic correlation in the diagnosis of oral lichen planus based on the presently available diagnostic criteria and suggestions for modifications. J Oral Pathol Med 32(9): 507-512.

7. Lichen planus. In: Mc Carthy PL, Shklar G (1980) Diseases of the oral mucosa. 2nd ed. Philadelphia: Lea and Febriger pp: 203-224.

8. Driann AJ, Fischman SL (1990) Controversies in oral medicine. Dental Clinics of North America 34(1): 159-169.

9. Verron J, Brightman (1994) Red and white lesions of the oral mucosa. In: Lynch MA, Brightman VJ, Greenberg MS, editors. Burket's oral medicinediagnosis and treatment ( $9^{\text {th }}$ edn.), Philadelphia: Lippincott-Raven pp: 99-111.

10. Immune-mediated disorders (2004) Contemporary oral and maxillofacial pathology ( $2^{\text {nd }}$ edn.). In: Sapp JP, Eversole LR, Wysocki GP (Eds.), Mosby, China pp. 257-262.

11. Scully C, Beyli M, Ferreiro MC, Ficarra G, Gill Y, et al. (1998) Update on Oral Lichen Planus: Etiopathogenesis and Management. Crit Rev Oral Biol Med 9(1): 86-122.

12. Lacy MF, Reade PC, Hay KD (1983) Lichen planus: A theory of pathogenesis. Oral surg 56(5): 521-526.

13. Eversole LR, Ringer M (1984) The role of dental restorative metals in the pathogenesis of oral lichen planus. Oral Surg 57: 383-387.

14. Bolewska J, Hansen HJ, Holmstrup P, Pindborg JJ, Stangerup M (1990) Oral mucosal lesions related to silver amalgam restorations. Oral Surg Oral Med Oral Pathol 70: 55-58.

15. Ostman PO, Anneroth G, Skoglund A (1996) Amalgam associated oral lichenoid reactions. Oral Surg Oral Med Oral Pathol Oral Radiol Endod 81: 459-465.

16. Hampf BGC, Malmstrom MJ, Aalberg VA, Hannula JA, Vikkula J (1987) Psychiatric disturbance in patients with oral lichen planus. Oral Surg Oral Med Oral Pathol 63: 429-432.

17. Silverman JS, Gorsky M, Lozada NF, Giannotti K (1991) A prospective study of findings and management in 214 patients with oral lichen planus. Oral Surg Oral Med Oral Pathol 72: 665-670.

18. Jontell M, Watts S, Wallstrom M, Levin L, Sloberg K (1990) Human pailloma virus in erosive oral lichen planus. J Oral Pathol Med 19: 273277.

19. Allen CM, Beck FM, Rossie KM, Kaul TJ (1986) Relation of stress and anxiety to oral lichen planus. Oral Surg Oral Med Oral Pathol 61: 44-46.

20. McCartan BE (1995) Psychological factors associated with oral lichen planus. J Oral Pathol Med 24: 273-275.

21. Lundstrom IMC (1983) Incidence of diabetes mellitus in patients with oral lichen planus. Int J Oral Surg 12: 147-152.

22. Sugerman PB, Savage NW, Walsh LJ, Zhao ZZ, Zhou XJ, et al. (2002) The Pathogenesis of oral lichen planus. Crit Rev Oral Biol Med 13(4): 350365 .

23. Walsh LJ, Savage NW, Ishii T, Seymour GJ (1990) Immunopathogenesis of oral lichen planus. J Oral Pathol Med 19(9): 389-396.

24. Zhao ZZ, Sugerman PB, Zhou XJ, Walsh LJ, Savage NW (2001) Mast cell degranulation and the role of T cell RANTES in oral lichen planus. Oral Dis 7: 246-251.

25. Dusek JJ, Frick WG (1982) Lichen planus: oral manifestations and suggested treatments. J Oral Maxillofac Surg 40(4): 240-244. 
26. Scully C, El-Kom M (1985) Lichen Planus. Review and update on pathogenesis. J Oral Pathol 14(6): 431-458.

27. Rajendran R (2006) Diseases of the skin. Shafer's text book of oral pathology ( $5^{\text {th }}$ edn.), Elsevier, India pp: 1103-1107.

28. Silverman S, Gorsky M, Lozada NF, Francisco S (1985) A prospective follow-up study of 570 patients with oral lichen planus: Persistence, remission, and malignant association. Oral Surg Oral Med Oral Pathol 60: $30-34$.

29. Tyldesley WR (1974) Oral lichen planus. Br J Oral Surg 11(3): 187-206.

30. Swan RH (1982) Oral lichen planus with associated nail changes. J Oral Med 37(1): 23-26.

31. Thorn JJ, Holmstrup P, Rindum J, Pindborg JJ (1988) Course of various clinical forms of oral lichen planus. A prospective follow-up study of 611patients.J Oral Pathol 17(5): 213-218.

32. Vinsent SD, Fotos PG, Baker KA, Williams TP (1990) Oral lichen planus: The clinical, histological, and therapeutic features of 100 cases. Oral Surg Oral Med Oral Pathol 70(2): 165-171.

33. Eisenberg E (1994) Clinicopathologic patterns of oral lichenoid lesions. In: Gold L editor. Surgical pathology: considerations in diagnosis and management. Oral and Maxillofacial Surgery Clinics of North America. 6(3): 445-463.

34. Chiappelli F, Kung MA, Nguyen P, Villanueva P, Farhadian EA, (1997) Cellular immune correlates of clinical severity in oral lichen planus: preliminary association with mood states. Oral Dis 3(2): 64-70.

35. Onofre MA, Sposto MR, Navarro CM, Motta ME, Turatti E, et al. (1997) Potentially malignant epithelial oral lesions: discrepancies between clinical and histological diagnosis. Oral Dis 3(3): 148-152.

36. Abbey LM, Kaugars GE, Gunsolley JC, Burns JC, Page DG, et al. (1995) Intraexaminer and interexaminer reliability in the diagnosis of oral epithelial dysplasia. Oral Surg Oral Med Oral Pathol Oral Radiol Endod 80(2): 188-191.
37. Karabulut A, Reibel J, Therkildsen MH, Praetorius F, Nielsen HW, et al. (1995) Observer variability in the histologic assessment of oral premalignant lesions. J Oral Pathol Med 24(5): 198- 200.

38. Toussaint S, Kamino H (1997) Noninfectious erythematous papular, and squamous diseases of the skin. In: Elder D, Elenitsas R, Jaworsky C, Johnson Jr. B, editors. Lever's histopthology of the skin. 8th ed. Philadelphia: Lippincott- Raven pp: 166-172.

39. Hedberg N, Ng A, Hunter N (1986) A semi-quantitative assessment of the histopathology of oral lichen planus. J Oral Pathol 15(5): 268-272.

40. Van der Meij EH, van der Wall I (2001) Granular cells in oral lichen planus. Oral Dis 7(2): 116-118.

41. Kaugars GE, Svirsky JA (1982) An update on the dyspastic/carcinomatous transformation of oral lichen planus. J Oral Med 37(3): 75-79.

42. Jong De WFB, Albert M, Banoczy J, Van Der Wall I (1984) Epithelial dysplasia in oral lichen planus. A preliminary report of a DutchHungarian study of 100 cases. Int J Oral Surg 13(3): 221-225.

43. Murti PR, Daftary DK, Bhonsle RB, Gupta PC, Mehta FS, et al. (1986) Malignant potential of oral lichen planus: observations in 722 patients from India. J Oral Pathol 15(2): 71-77.

44. Laskaris G, Sklavounou A, Angelopoulos A (1982) Direct immunofluorescence in oral lichen planus. Oral Surg 53 (5): 483-487.

45. Schiodt M, Holmstrup P, Dabelsteen E, Ullman S (1981) Deposits of immunoglobulins, complement, and fibrinogen in oral lupus erythematosus, lichen planus, and leukoplakia. Oral Surg 51(6): 603608.

46. Lamey PJ, McCartan BE, MacDonald DG, MacKie RM (1995) Basal cell cytoplasmic autoantibodies in oral lichenoid reactions. Oral Surg Oral Med Oral Pathol Oral Radiol Endod 79(1): 44-49.

47. Lodi G, Scully C, Carrozzo M, Griffiths M, Sugerman, Thongprasom K (2007) Oral Surg Oral Med Oral Pathol Oral Radiol Endod 103(suppl 1): S25.e1-S25.

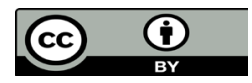

This work is licensed under Creative Commons Attribution 4.0 License

Submission Link: https://biomedres.us/submit-manuscript.php

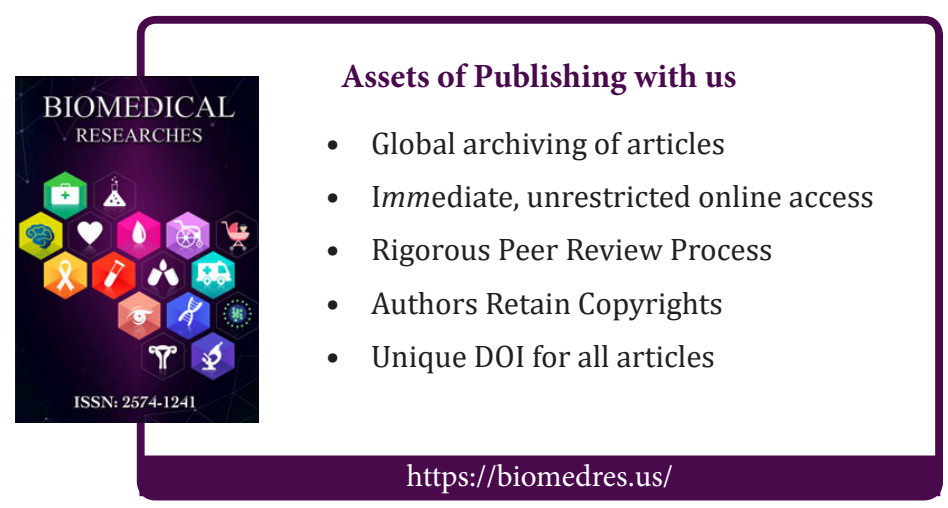

